\title{
Parametric numerical solutions of additive manufacturing processes
}

Cite as: AIP Conference Proceedings 2113, 100007 (2019); https://doi.org/10.1063/1.5112640

Published Online: 02 July 2019

Giacomo Quaranta, Eberhard Haug, Jean Louis Duval, Elias Cueto, and Francisco Chinesta

ARTICLES YOU MAY BE INTERESTED IN

Data-driven correction of models for deformable solids

AIP Conference Proceedings 2113, 100010 (2019); https://doi.org/10.1063/1.5112643

Towards parametric RTM processes: The interpolative mapping

AIP Conference Proceedings 2113, 100004 (2019); https://doi.org/10.1063/1.5112637

Model reduction method for the simulation of the selective laser melting process

AIP Conference Proceedings 2113, 150008 (2019); https://doi.org/10.1063/1.5112684

\section{Conference Proceedings}

Get $30 \%$ off all

Enter Promotion Code $P D F 30$ at checkout print proceedings! 


\title{
Parametric Numerical Solutions of Additive Manufacturing Processes
}

\author{
Giacomo Quaranta $^{1, b)}$, Eberhard Haug ${ }^{1, \mathrm{c})}$, Jean Louis Duval ${ }^{1, \mathrm{~d})}$, Elias Cueto ${ }^{2, \mathrm{e})}$ and \\ Francisco Chinesta ${ }^{3,1, a), f)}$ \\ ${ }^{1}$ ESI Group, 3bis rue Saarinen, 94528 Rungis CEDEX, France. \\ ${ }^{2}$ Aragon Institute of Engineering Research. Universidad de Zaragoza, Maria de Luna s/n, 50018 Zaragoza, Spain . \\ ${ }^{3}$ ENSAM ParisTech, 151 rue de l'Hôpital. 75013 Paris, France.

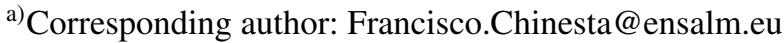 \\ b) Giacomo.Quaranta@esi-group.com \\ ${ }^{c}$ Eberhard.Haug@esi-group.com \\ d) Jean-Louis.Duval@esi-group.co \\ e)ecueto@unizar.es \\ ${ }^{\text {f)}}$ Francisco.Chinesta@ensam.eu
}

\begin{abstract}
Additive manufacturing is the more and more considered in industry, however efficient simulation tools able to perform accurate predictions are still quite limited. The main difficulties for an efficient simulation are related to the multiple scales, the multiple and complex physics involved, as well as the strong dependency on the process trajectory. In [21] authors proposed the use of advanced model reduction techniques for performing parametric simulations of additive manufacturing processes, where deposition trajectory, the intensity of the thermal shrinkage and the deposited layers were considered as model parameters. The resulting simulation tool allowed evaluating in real-time the impact of the parameters just referred on the part distortion, and proceed to the required geometrical compensation. In the present work we address the use of that parametric solution with three different purposes: (i) evaluating the parameters leading to the minimal part distortion; (ii) evaluating the solution sensitivity to the different parameters, and in particular to the ones related to the deposition trajectory; and (iii) propagating the uncertainty related to the intensity of the thermal shrinkage.
\end{abstract}

\section{Introduction}

The adoption of Additive Manufacturing (AM) technologies at the industrial level has set out several challenges for the scientific community, ranging from the study of the interactions at the scale of the particle to the prediction of the mechanical state of the final part. A global process modeling and its associated efficient solution remain nowadays out of reach because many challenges persist, concerning the multiple physics and scales requiring fine and rich models, well calibrated in extreme conditions (in space, time and process conditions). These circumstances and the extremely long process trajectory length compromise the efficiency of well experienced numerical simulations tools. These difficulties can be grouped in three categories:

- Geometric complexity. The flexibility of AM technologies allows for the use of topology optimization in the design stage. This results typically in thin parts, very sensitive to distortions. Complex geometries also require finer meshes and hence, large size numerical models.

- Strong dependence on the fabrication strategy. This is mainly due to the non-linear and coupled models involved in phase transformations and inelastic behaviors.

- The incremental nature of the process. A part is composed of thousands of deposited layers each of them constituted of a succession of tapes less than $1 \mathrm{~mm}$ thick. 
These difficulties justify the fact that standard approaches (e.g. finite elements combined with the so-called borndead-elements technique [12]) lead to prohibitive simulation times. Thus, well experienced simulation technologies cannot be used for performing parametric analysis and efficiently exploring the parametric design space for optimizing processes or for performing simulation-based real-time control.

Many works focused on the modeling and simulation of additive manufacturing, among them $[3,10,11,13,14$, $15,16,17,18,19,20,22]$. One of the main obstacles to an efficient simulation of AM processes for predicting part distortions, independently of the model richness, is related to the numerical model itself, by the fact of assembling and solving very large systems of equations at each time step and in a geometry that is evolving in time [1]. Thus, reduced order modeling was envisaged as a possible remedy for attaining faster simulations without compromising the accuracy. A possible alternative within the MOR framework consists in extracting "offline" the most significant modes involved in the model solution, that will constitute the so-called reduced approximation basis, and then project the solution of "quite similar" problems in that reduced basis. Because the number of functions involved in the reduced basis is quite low, only few coefficients must be calculated for determining the problem solution. Consequently, a discrete problem of very small size must be solved at each iteration or time step. Thus, MOR-based discretization techniques allow important computing-time savings, of several orders of magnitude in some cases, making possible accommodating to real-time constraints [7, 9]. Another MOR-based route, close to the previous one, considered in our works, consists of calculating offline using all the needed computational resources, e.g. HPC, and computing time, a parametric solution containing the solution of all possible scenarios, that is then particularized online using light computational facilities, as deployed devices, tablets or even smartphones, for performing efficient simulation, optimization, inverse analysis, uncertainty propagation and simulation-based control, all them under real-time constraints. This was the solution retained in many applications where the so-called PGD - Proper Generalized Decomposition - was able to compute parametric solutions of models representing complex processes, structures and systems $[4,5,6,9,2,8]$. Thus, few hours of intensive offline computation allow the construction of very general parametric solutions able to ensure almost instantaneous responses to queries, while keeping the level of accuracy of usual discretizations.

\section{PROCESS PARAMETRIZATION}

\section{Parametrizing deposition trajectories}

In [21] we proposed an approach that consists of associating the trajectories to the iso-field curves $\phi=$ cte related to a certain parametrized partial differential equation involving the scalar field $\phi(x, y)$. For that purpose we proposed considering the Poisson equation defined in the rectangular domain $\Omega=(0, L) \times(0, H)$

$$
\Delta \phi(x, y)=s(x, y),
$$

with the parametrized Dirichlet boundary conditions

$$
\left\{\begin{array}{l}
\phi(x, y=0)=\mu_{1}+\left(\mu_{2}-\mu_{1}\right) \frac{x}{L} \\
\phi(x, y=H)=\mu_{4}+\left(\mu_{4}-\mu_{3}\right) \frac{x}{L} \\
\phi(x=0, y)=\mu_{1}+\left(\mu_{4}-\mu_{1}\right) \frac{y}{H} \\
\phi(x=L, y)=\mu_{2}+\left(\mu_{3}-\mu_{2}\right) \frac{y}{H}
\end{array},\right.
$$

that represent linear variations on each rectangle side, parametrized by the values at the corners $\phi(0,0)=\mu_{1}, \phi(L, 0)=$ $\mu_{2}, \phi(L, H)=\mu_{3}$ and $\phi(0, H)=\mu_{4}$. When all these parameters defining the boundary condition vanish, and the source term takes a unit value, i.e. $s(x, y)=1$, the solution iso-curves are expected representing concentric trajectories.

The parametric solution of this problem is easily computable because of its linearity, and consequently the use of the standard constructor of PGD parametric solutions is not compulsory. One must simply solve the following five boundary value problems, the first four related to the enforced Dirichlet boundary conditions,

$$
\left\{\begin{array}{l}
\Delta \phi^{1}(x, y)=s(x, y), \text { with } s(x, y)=0 \& \mu_{1}=1, \mu_{2}=\mu_{3}=\mu_{4}=0 \\
\Delta \phi^{2}(x, y)=s(x, y), \text { with } s(x, y)=0 \& \mu_{2}=1, \mu_{1}=\mu_{3}=\mu_{4}=0 \\
\Delta \phi^{3}(x, y)=s(x, y), \text { with } s(x, y)=0 \& \mu_{3}=1, \mu_{1}=\mu_{2}=\mu_{4}=0 \\
\Delta \phi^{4}(x, y)=s(x, y), \text { with } s(x, y)=0 \& \mu_{4}=1, \mu_{1}=\mu_{2}=\mu_{3}=0
\end{array},\right.
$$


and the last the source term $s(x, y)$

$$
\Delta \phi^{5}(x, y)=s(x, y), \text { with } s(x, y)=1 \& \mu_{1}=\mu_{2}=\mu_{3}=\mu_{4}=0 .
$$

Now, any other solution for any choice of parameters $\mu_{1}, \cdots, \mu_{4}$ and $\mu_{5}=s(x, y)$ can be obtained from

$$
\phi\left(x, y, \mu_{1}, \cdots, \mu_{5}\right)=\mu_{1} \phi^{1}(x, y)+\cdots+\mu_{5} \phi^{5}(x, y) .
$$

\section{A simplified parametric thermo-mechanical model}

In [21] among the numerous possibilities a wired model was considered, with the thermal shrinkage following the deposition trajectory. Thus, in the local system of coordinates, shrinkage stresses are expected having the expression

$$
\tilde{\boldsymbol{\sigma}}_{0}(\mathbf{P})=\left(\begin{array}{lll}
\lambda & 0 & 0 \\
0 & 0 & 0 \\
0 & 0 & 0
\end{array}\right),
$$

where the shrinkage intensity $\lambda$ will be introduced as an model extra-parameter.

The global equilibrium requires expressing shrinkage stresses, given at each point by expression (6) in the local coordinate system related to the local trajectory, in the global coordinate systems $(x, y, z)$. For that purpose we should consider the rotation of it according to

$$
\sigma_{0}(\mathbf{P})=\mathbf{R}^{T} \tilde{\boldsymbol{\sigma}}_{0}(\mathbf{P}) \mathbf{R}
$$

with $\mathbf{R}$ the rotation matrix. In the model just described, shrinkage stresses depend parametrically on the trajectory (that controls its anisotropy), and consequently when inserted into the virtual work variational principle, it results in a parametric displacement field representing the part distortion: $\mathbf{u}\left(\mathbf{x}, \mu_{1}, \cdots, \mu_{5}, \lambda\right)$.

\section{NUMERICAL RESULTS}

In this section we consider the manufacturing process of a part, where deposition trajectory, the intensity of the thermal shrinkage and the deposited layers were considered as model parameters. The resulting simulation tool allows evaluating in real-time the impact of the parameters just referred on the part distortion. Moreover, that parametric solution is used here with three different purposes: (i) evaluating the parameters leading to the minimal part distortion; (ii) evaluating the solution sensitivity to the different parameters, and in particular to the ones related to the deposition trajectory; and (iii) propagating the uncertainty related to the intensity of the thermal shrinkage.

Figs. 1 depicts the deformed geometry for two different values of the thermal shrinkage intensity, where it can be noticed that distortions vanish when $\lambda$ (referred as $L$ in the GUI application) vanishes and increases with it. The parameters leading to null derivatives of the parametric displacement with respect to parameters $\mu_{i}, i=1, \cdots, 5$ defining the deposition trajectory, defined the trajectory minimizing part distortion. That optimal solution is depicted in Fig. 2. Figure 3 depicts the derivative of the displacement with respect to parameters $\mu_{1}$ and $\mu_{5}$ for the parameters related to the optimal process leading to the part shown in Fig. 2. Finally, if the thermal shrinkage intensity $\lambda$ is assumed normally distributed (Fig. 4 (left)), the norm of the distortion becomes distributed as depicted in Fig. 4(right).

\section{CONCLUSIONS}

This paper presented a simplified parametric modeling of distortions occurring in parts manufactured by additive manufacturing. After having introduced a parametrization of the process trajectories as the iso-values of a parametrized partial differential equation, thermal shrinkage was expressed parametrically with respect to the process trajectory. Finally two new parameters were introduced, the first controlling the shrinkage intensity and the second the process progression, that is, the number of deposited layers. The parametric solution was introduced into a GUI application able to evaluate the effect of any parameter in almost real-time. Moreover that parametric solution was also successfully used with other three different purposes: (i) evaluating the parameters leading to the minimal part distortion; (ii) evaluating the solution sensitivity to the different parameters, and in particular to the ones related to the deposition trajectory; and (iii) propagating the uncertainty related to the intensity of the thermal shrinkage. 

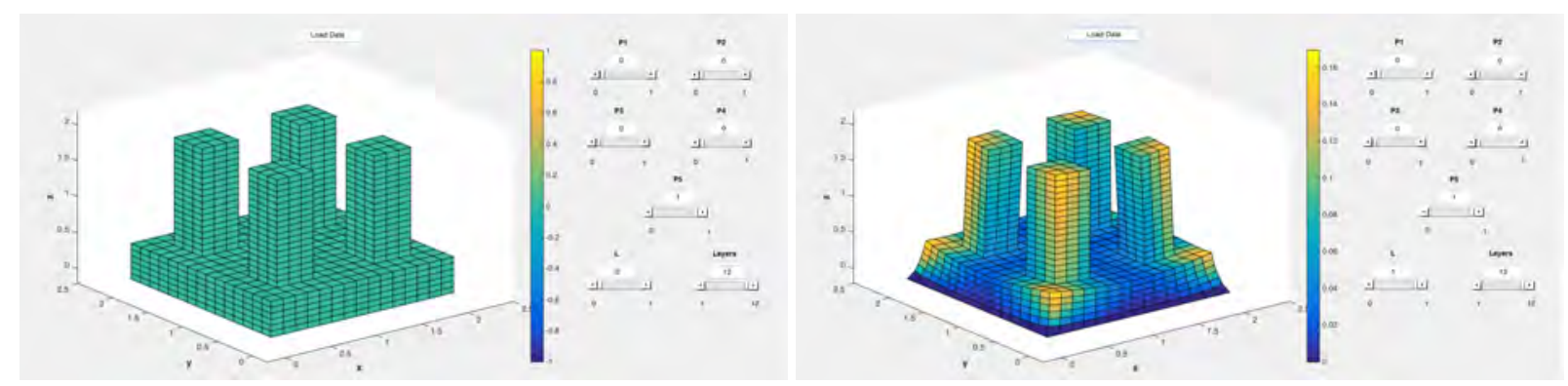

FIGURE 1. Parametric distortions for $\mu_{1}=0, \mu_{2}=0, \mu_{3}=0, \mu_{4}=0, \mu_{5}=1$ : (left) $L=\lambda=0$ and (right) $L=\lambda=1$ (Dimensions: $\times 10 \mathrm{~cm}$. Displacements in $\mathrm{cm}$.)

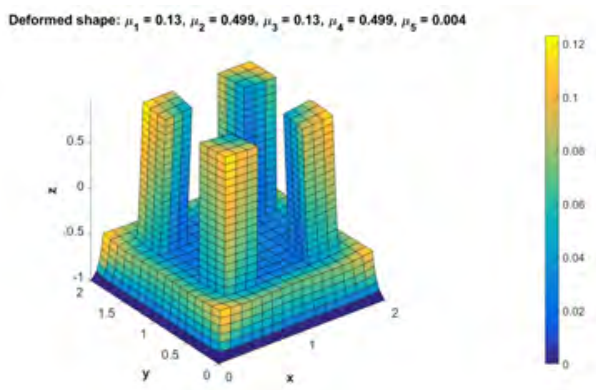

FIGURE 2. Optimal process minimizing part distortion
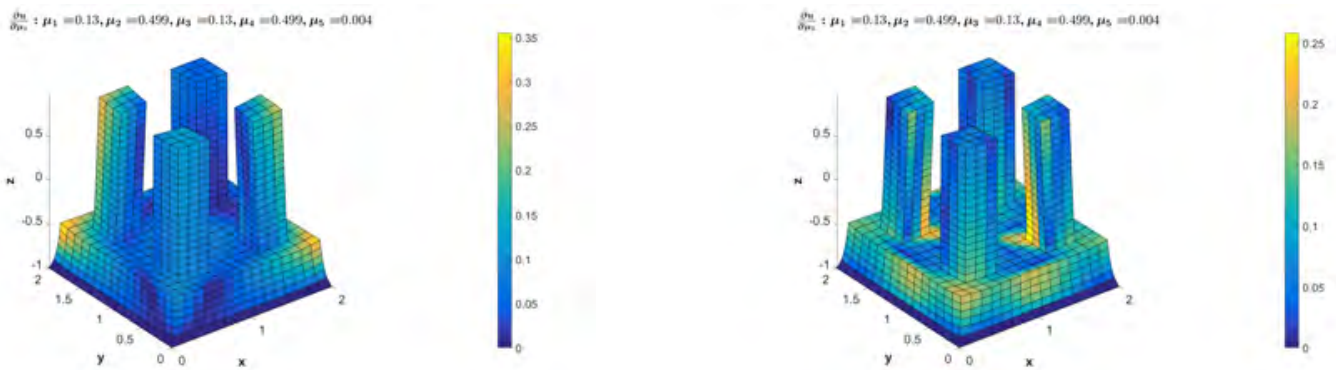

FIGURE 3. Sensitivity analysis: Displacement derivative with respect (left) parameter $\mu_{1}$ and $\mu_{5}$ (right)
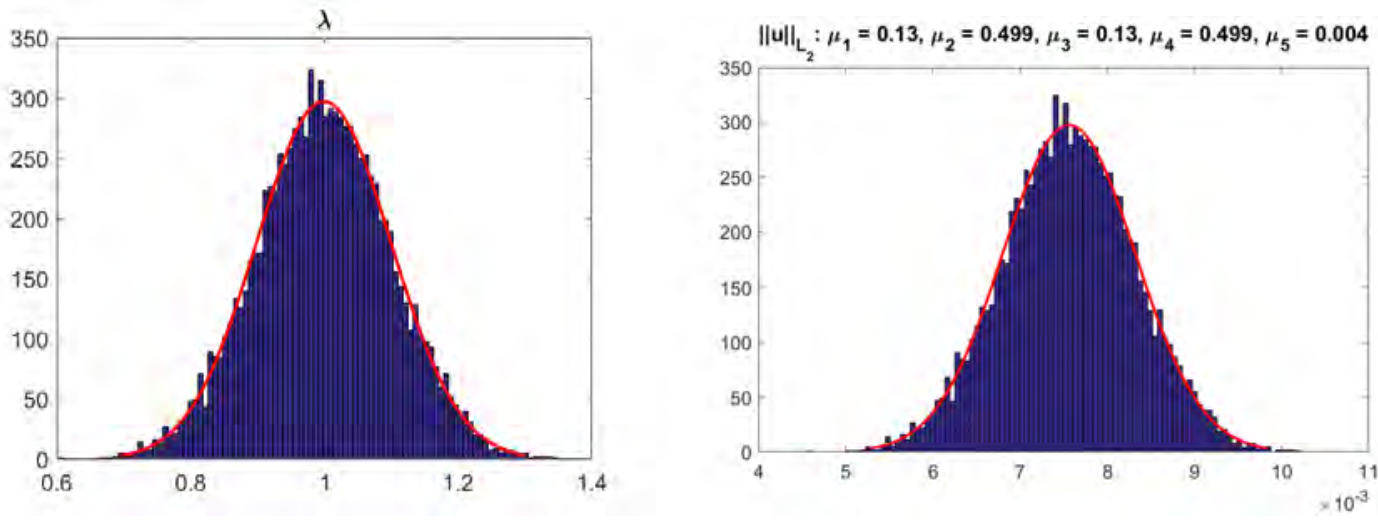

FIGURE 4. Uncertainty propagation related to the thermal shrinkage 


\section{REFERENCES}

[1] J.V. Aguado, B. Bognet, D. Canales, O. Desmaison, F. Boitout, F. Chinesta. A reduced order modeling approach for fast thermo-mechanics simulation of additive layer manufacturing. ECCOMAS Conference on Simulation for Additive Manufacturing, Munich, Germany, 2017.

[2] N. Bur, P. Joyot, C. Ghnatios, P. Villon, E. Cueto, F. Chinesta. On the use of model order reduction for simulating automated fibre placement processes. Advanced Modeling and Simulation in Engineering Sciences, 3:4, 2016

[3] T. Chen, Y. Zhang, Yuwen. Numerical Simulation of Two-Dimensional Melting and resolidification of a Two-Component Metal Powder Layer in Selective Laser Sintering Process. Numerical Heat Transfer, Part A: Applications, 46:7, 633-649, 2004.

[4] F. Chinesta, P. Ladeveze, E. Cueto. A short review in model order reduction based on proper generalized decomposition. Arch. Comput. Methods Eng., 18, 395-404, 2011.

[5] F. Chinesta,A. Leygue, F. Bordeu, J.V. Aguado, E. Cueto, D. Gonzalez, I. Alfaro, A. Ammar, A. Huerta. PGD-based computational vademecum for efficient design, optimization and control. Arch. Comput. Methods Eng., 20, 31-59, 2013.

[6] F. Chinesta, R. Keunings, A. Leygue. The Proper Generalized Decomposition for Advanced Numerical Simulations. A primer. Springerbriefs, Springer, 2014.

[7] F. Chinesta, P. Ladeveze (Edts). Separated Representations and PGD Based Model Reduction: Fundamentals and Applications. CISM-Springer, 2014.

[8] F. Chinesta, A. Leygue, B. Bognet, C. Ghnatios, F. Poulhaon, F. Bordeu, A. Barasinski, A. Poitou, S. Chatel, S. Maison-Le-Poec. First steps towards an advanced simulation of composites manufacturing by automated tape placement. International Journal of Material Forming, 7, 81-92, 2014.

[9] F. Chinesta, A. Huerta, G. Rozza, K. Willcox. Model Order Reduction. Chapter in the Encyclopedia of Computational Mechanics, Second Edition, Erwin Stein, René de Borst \& Tom Hughes Edt., John Wiley \& Sons Ltd., 2015.

[10] M. Chiumenti, M. Cervera, A. Salmi, C. Agelet de Saracibar, N. Dialami, K. Matsui. Finite element modeling of multi-pass welding and shaped metal deposition processes. Computer Methods in Applied Mechanics and Engineering, 199:37, 2343-2359, 2010.

[11] M. Chiumenti, X. Lin, M. Cervera, W. Lei, Y. Zheng, W. Huang. Numerical simulation and experimental calibration of Additive Manufacturing by blown powder technology. Part I: thermal analysis. Rapid Prototyping Journal, 2017 (In press).

[12] J. Ding, P. Colegrove, J. Mehnen, P.M. Sequeira Almeida, F. Wang, S. Williams. Thermomechanical analysis of Wire and Arc Additive Layer Manufacturing process on large multi-layer parts. Computational Materials Science, 50, 3315-3322, 2011.

[13] L. Dong, A. Makradi, S. Ahzi, Y. Remond. Three-dimensional transient finite element analysis of the selective laser sintering process. Journal of Materials Processing Technology, 209:2, 700-706, 2009.

[14] J.C. Heigel, P. Michaleris, E.W. Reutzel. Thermo-mechanical model development and validation of directed energy deposition additive manufacturing of Ti-6Al-4V. Additive Manufacturing, 5, 9-19, 2015.

[15] C. Korner, E. Attar, P. Heinl. Mesoscopic simulation of selective beam melting processes. Journal of Materials Processing Technology, 211:6, 978-987, 2011.

[16] C. Korner, A. Bauereis, E. Attar. Fundamental consolidation mechanisms during selective beam melting of powders. Modelling and Simulation in Materials Science and Engineering, 21:8, 085011, 2013.

[17] S. Kolossov, E. Boillat, R. Glardon, P. Fischer, M. Locher. 3D FE simulation for temperature evolution in the selective laser sintering process. International Journal of Machine Tools and Manufacture, 44:2, 117-123, 2004.

[18] M. Labudovic, D. Hu, R. Kovacevic. A three dimensional model for direct laser metal powder deposition and rapid prototyping. Journal of Materials Science, 38, 35-49, 2003.

[19] Y. Li, D. Gu. Parametric analysis of thermal behavior during selective laser melting additive manufacturing of aluminum alloy powder, Materials \& Design, 63, 856-867, 2014. 
[20] S. Marimuthu, D. Clark, J. Allen, A.M. Kamara, P. Mativenga, L. Li, R. Scudamore. Finite element modelling of substrate thermal distortion in direct laser additive manufacture of an aero-engine component. Proceedings of the Institution of Mechanical Engineers, Part C: Journal of Mechanical Engineering Science, 227:9, 1987-1999, 2013.

[21] G. Quaranta, E. Haug, J.L. Duval, F. Chinesta. Parametric evaluation of part distortion in additive manufacturing processes. International Journal of Material Forming. In press, 2018.

[22] K. Zeng, D. Pal, N. Patil, and B.E. Stucker. A new dynamic mesh method applied to the simulation of selective laser melting. Proceedings of the Solid Freeform Fabrication Symposium, Austin, TX, Aug. 1214, 549-559, 2013. 